\title{
Coplanar and coaxial orientations of RNA bases and helices
}

\author{
ALAIN LAEDERACH, ${ }^{1,3}$ JOSEPH M. CHAN, ${ }^{1,3}$ ARMIN SCHWARTZMAN, ${ }^{2}$ ERIC WILLGOHS, ${ }^{1}$ and \\ RUSS B. ALTMAN ${ }^{1}$ \\ ${ }^{1}$ Department of Genetics, Stanford University, Stanford, California 94305, USA \\ ${ }^{2}$ Department of Biostatistics, Harvard School of Public Health and Dana-Farber Cancer Institute, Boston Massachusetts 02115, USA
}

\begin{abstract}
Electrostatic interactions, base-pairing, and especially base-stacking dominate RNA three-dimensional structures. In an A-form RNA helix, base-stacking results in nearly perfect parallel orientations of all bases in the helix. Interestingly, when an RNA structure containing multiple helices is visualized at the atomic level, it is often possible to find an orientation such that only the edges of most bases are visible. This suggests that a general aspect of higher level RNA structure is a coplanar arrangement of base-normal vectors. We have analyzed all solved RNA crystal structures to determine the degree to which RNA base-normal vectors are globally coplanar. Using a statistical test based on the Watson-Girdle distribution, we determined that 330 out of 331 known RNA structures show statistically significant $(p<0.05$; false discovery rate $[$ FDR] $=0.05)$ coplanar normal vector orientations. Not surprisingly, $94 \%$ of the helices in RNA show bipolar arrangements of their base-normal vectors $(p<0.05)$. This allows us to compute a mean axis for each helix and compare their orientations within an RNA structure. This analysis revealed that $62 \%(208 / 331)$ of the RNA structures exhibit statistically significant coaxial packing of helices $(p<0.05$, FDR $=$ 0.08). Further analysis reveals that the bases in hairpin loops and junctions are also generally planar. This work demonstrates coplanar base orientation and coaxial helix packing as an emergent behavior of RNA structure and may be useful as a structural modeling constraint.
\end{abstract}

Keywords: RNA; structure; planar; bipolar; coaxial

\section{INTRODUCTION}

RNA adopts well-defined three-dimensional structures that carry out specific functions in the cell such as splicing (Cech 1990; Schroeder et al. 2004) or gene regulation (Tucker and Breaker 2005). The defining characteristics of RNA structure is base pairing and base stacking (Cate et al. 1996). The most common structural element in RNA is the A-form helix that consists of sequentially stacked WatsonCrick base pairs (Lemieux and Major 2002; Lescoute et al. 2005; Leontis et al. 2006b). Other structural elements include hairpin loops, (two-, three-, and four-way) junctions, and single-stranded regions (Lemieux and Major 2002; Lescoute et al. 2005; Leontis et al. 2006b). Elements of secondary

\footnotetext{
${ }^{3}$ These authors contributed equally to this work.

Reprint requests to: Russ B. Altman, Department of Genetics, Stanford Medical Center, 300 Pasteur Drive, Lane L301, Mail Code 5120, Stanford University, Stanford, California 94305, USA; e-mail: russ.altman@ stanford.edu; fax: (650) 725-3863.

Article published online ahead of print. Article and publication date are at http://www.rnajournal.org/cgi/doi/10.1261/rna.381407.
}

structure are stabilized by highly specific tertiary contacts, which define the three-dimensional structure of the RNA (Leontis et al. 2002a).

Much attention has been placed on defining structural motifs in RNA (Leontis et al. 2002a,b, 2006a; Leontis and Westhof 2003; Lescoute et al. 2005). These are specific threedimensional arrangements of 3-15 nucleotides (nt) that occur multiple times in one or many structures (Lescoute et al. 2005; Leontis et al. 2006b). This detailed atomic description is extremely valuable for understanding RNA structure, as well as predicting it (Leontis et al. 2006a). In this article, however, we focus on a global phenomenon in RNA structure, the relative orientation of bases.

To best visualize an RNA structure, it is common practice to rotate it in a three-dimensional molecular visualization program (Koradi et al. 1996). We noted that the most visually appealing orientation for an RNA molecule is often one in which the edges of the maximum number of bases are visible, such that one can see through most of the molecule. This is illustrated in Figure 1, A and B for the classic tRNA and P4-P6 subdomain structures, respectively 
A

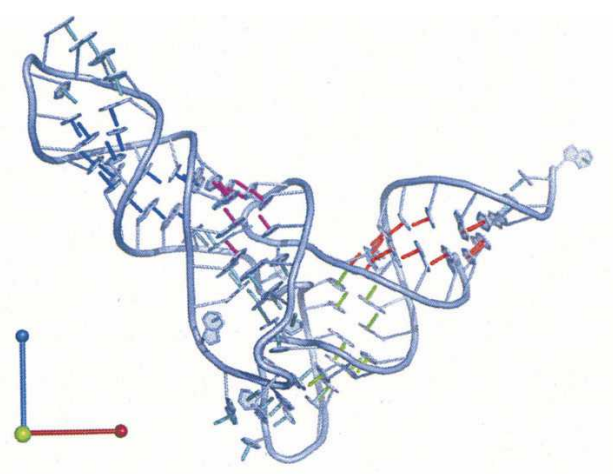

C

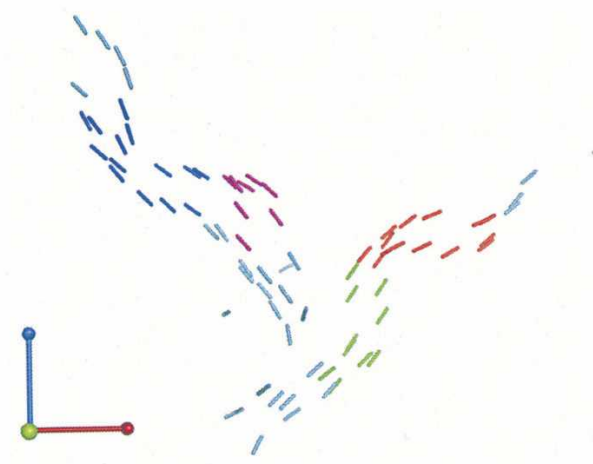

E

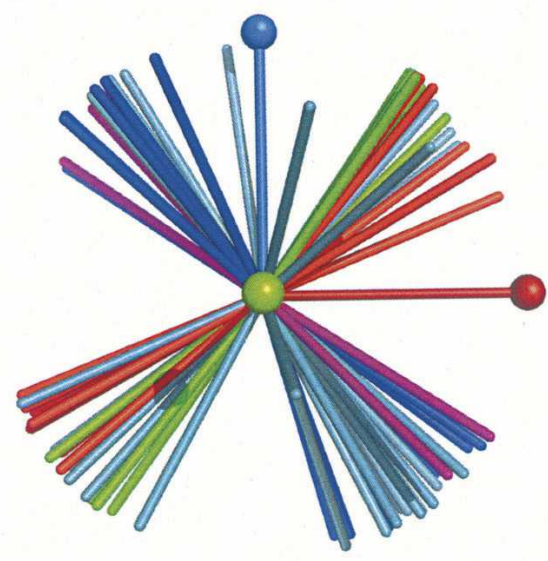

B

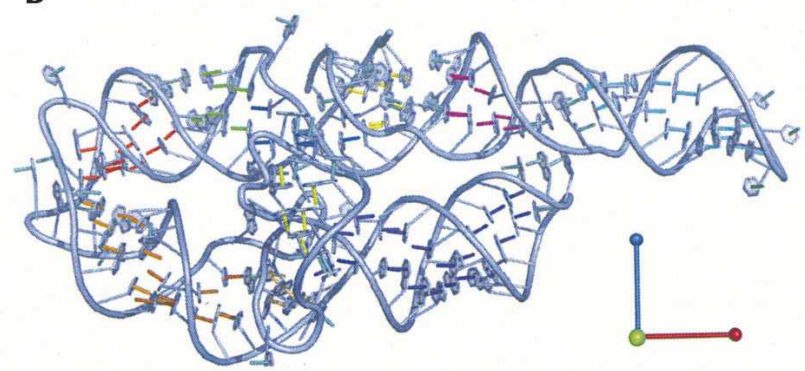

D

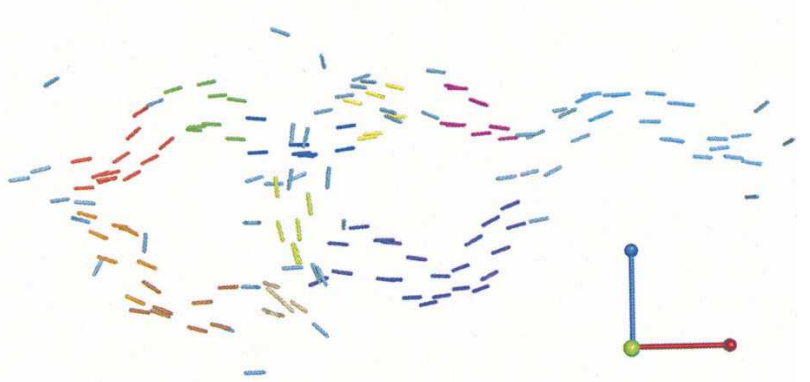

$\mathbf{F}$

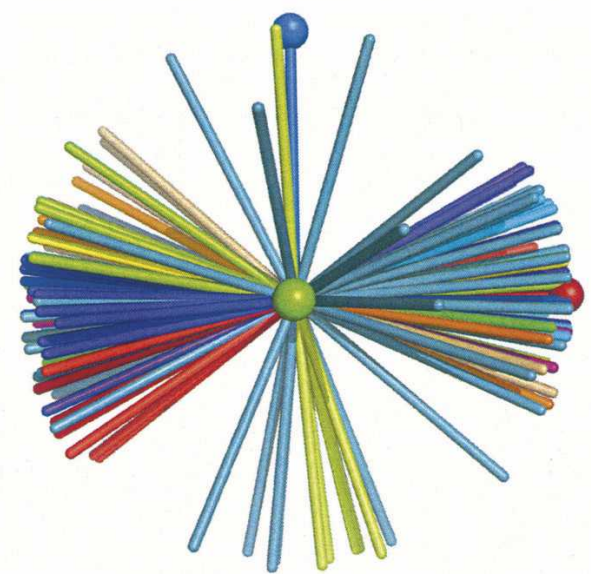

FIGURE 1. $(A, B)$ Structural representations of the phe-tRNA (PDB ID 1EHZ) and P4-P6 subdomain of the L-21 T. thermophila group I intron (PDB ID 1GID) crystal structures, respectively. Base-normal vectors are drawn and colored according to helix affiliation. (C,D) Illustrations of base-normal vectors with RNA structural representation not shown. (E,F) Same as above, but all base-normal helices have been translated to the origin while maintaining the same orientation. The three eigenvectors $\left(\lambda_{1}, \lambda_{2}\right.$, and $\left.\lambda_{3}\right)$ are rendered as red, blue, and green vectors with spherical tips, respectively. In all three representations the same frame of reference is maintained. This article reports on the statistical tests of the coplanar and bipolar nature of RNA base-normal vectors.

(Cate et al. 1996). In fact, we chose the orientation of these molecules based on an eigenvalue decomposition of the scatter matrix of the base-normal vectors described below. The normal vectors to each base are drawn in Figure 1, C and $\mathrm{D}$ and from this illustration it becomes apparent that most of these vectors are coplanar. This is also apparent in Figure 1, E and $\mathrm{F}$ where the vectors are translated to the origin while maintaining their orientation. Coaxial arrangements of helices have already been suggested as an emergent behavior in RNA structure (Leontis et al. 1995;
Duckett et al. 1997). However, the general nature of the coaxial orientation of RNA helices has yet to be quantitatively defined for all known RNA structures. A significant number of large RNA structures have now been solved crystallographically and provide a suitable data set for statistical analysis of RNA structure.

We develop a mathematical formalism based on tools from directional statistics to evaluate the degree of coplanarity in all known RNA structures. We aim to determine whether global coplanarity of bases is a statistically significant 
emergent behavior of RNA structure. Furthermore, we evaluate whether this is due to the dominance of helices in RNA structure or whether there is a general preference for helices to pack coaxially. Finally, we evaluate the statistical significance of coplanarity in the individual structural elements of RNA such as junctions, hairpins, and single-stranded regions.

\section{RESULTS}

Our analysis of RNA structure is based on the ensemble of normal vectors for each individual structure, translated to the origin as illustrated in Figure 1, E and F. We consider in this article three types of distributions of the directions of vectors. In Figure $2 \mathrm{~A}$, we illustrate a perfectly random distribution of vector directions, while in Figure $2 \mathrm{~B}$ we illustrate a coplanar distribution, and in Figure 2C a bipolar distribution. We consider for each RNA structure the degree to which the directions of the base-normal vectors fit a random, bipolar, or coplanar distribution.

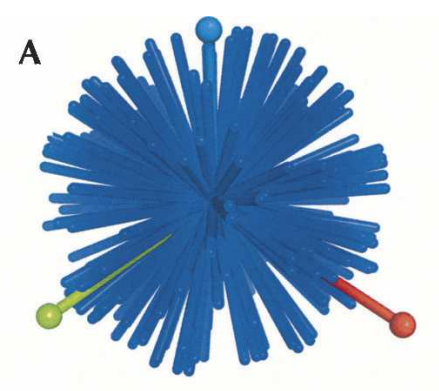

B
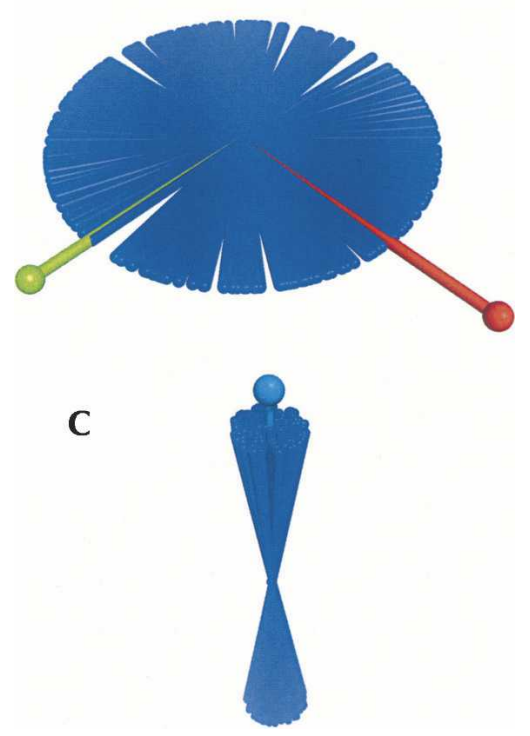

FIGURE 2. Illustrations of vector distributions considered in the article. Vectors are drawn as blue lines, and the three eigenvectors of each distribution are illustrated as red, green, and cyan vectors with a ball tip. $(A-C)$ Random, coplanar, and bipolar distributions, respectively.
As described in detail in the Materials and Methods section, we use a mathematical formalism that describes each distribution in terms of the contributions of three parameters: $c_{b}, c_{p}$, and $c_{r}$ (i.e., bipolar, planar, and random) (Zhang et al. 2004). Since the three parameters add up to one, it is appropriate to plot them on barycentric coordinates as illustrated in Figure 3. The three vertices of the triangle represent a pure contribution of one of the three parameters. In Figure 3, the top vertex represents $c_{r}$ (or a random distribution), while the left and right vertices correspond to $c_{b}$ and $c_{p}$ (bipolar and coplanar), respectively. If we now consider a distribution of normal vectors based on a solved RNA crystal structure, we can determine the three parameters and plot them on this graph. For example, the P4-P6 subdomain of the Thermus thermophila group I intron is plotted as a cyan star in Figure 3. The star is near the bottom left vertex of the graph, indicating that the distributions of normal vectors are mostly bipolar, which is in agreement with Figure 1E. In the following sections, we repeat this analysis for all RNAs of known structure and furthermore develop a statistical test that allows us to determine significant coplanarity and/or bipolarity in distributions.

\section{Statistical analysis of base-normal vectors}

Three hundred thirty-one RNA crystal structures fit our selection criteria in the most recent release of the Nucleic Acid Database (Berman et al. 2003). For each RNA structure, the base-normal vectors were computed as illustrated in Figure 1, and the barycentric coordinates determined as described in Materials and Methods. Figure 3 illustrates the results of this basic analysis and reveals that most RNA molecules show either coplanar or bipolar arrangements of their base-normal vectors. Furthermore, most tRNAs (plotted in red) exhibit a clear coplanar arrangement of their base-normal vectors in agreement with the high degree of structural conservation in this family of RNAs (Auffinger and Westhof 2001).

The one class of RNAs that does not appear to present a coplanar or bipolar arrangement of base-normal helices is the ribosomal RNAs (plotted in green). Most ribosomal RNAs appear near the top of the triangle in Figure 3. The one drawback of visualizing the results of our structural analysis in barycentric coordinates is that it does not take into account the size of (and number of bases in) the RNA molecule. It is therefore difficult to evaluate if the positions of the ribosomal RNAs are statistically different from a random orientation of base-normal vectors. We therefore performed a statistical test of coplanarity of the basenormal vectors as described in the Materials and Methods section. The results of this test are summarized in Figure 4A. The solid line represents the mean angular dispersion with respect to the best-fitting plane of a random spherical distribution as a function of $n$, obtained by simulation. 


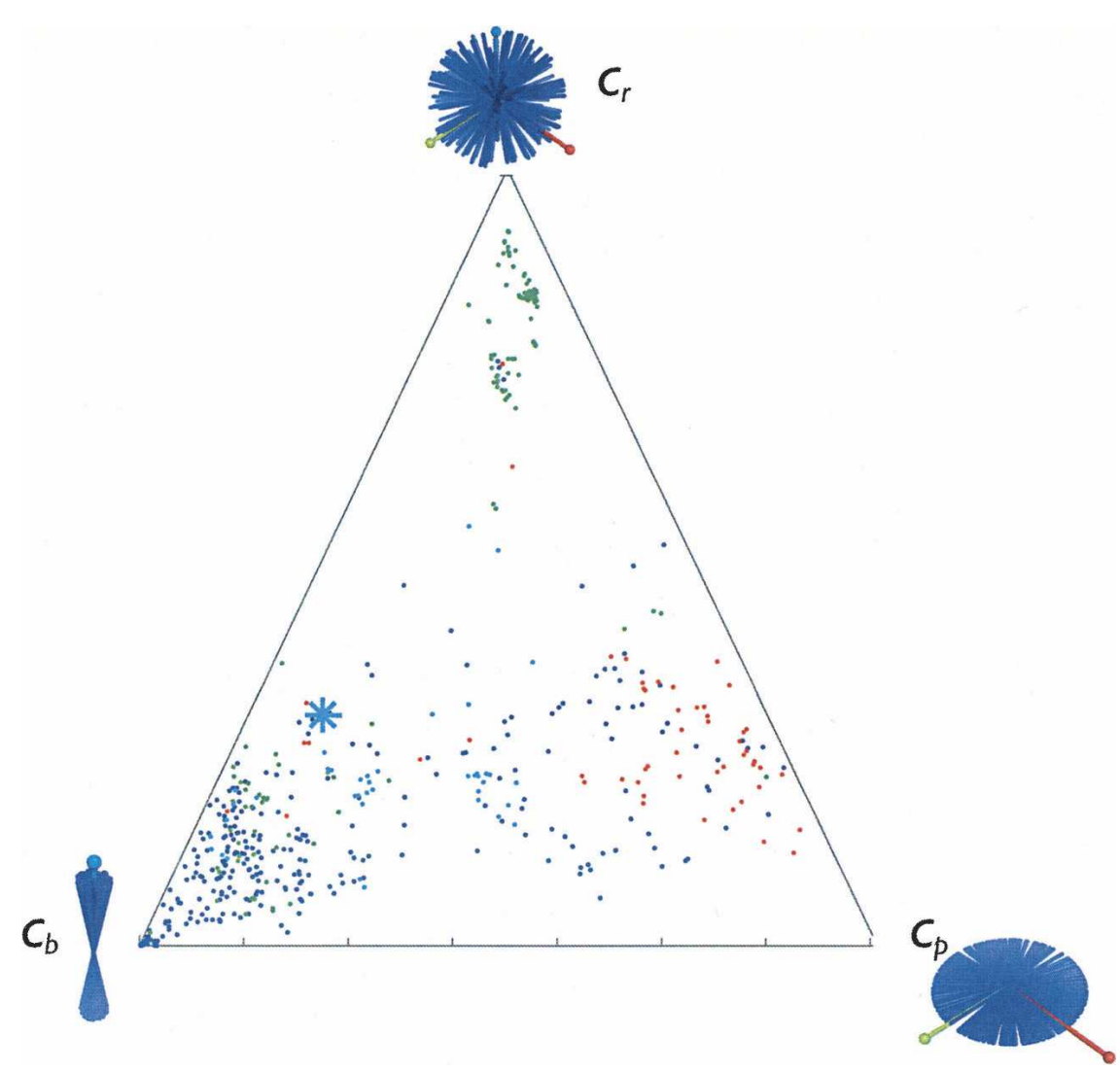

FIGURE 3. Plot in barycentric coordinates for all known RNA structures as defined by Equation (2). Each dot represents a single, known RNA structure. The position of the dot indicates the bipolar (bottom left), coplanar (bottom right), or random (top) nature of the distribution of base-normal vectors in the RNA molecule. The cyan star indicates the corresponding barycentric coordinates of the P4-P6 subdomain of the L-21 T. thermophila group I intron crystal structure. Red, green, cyan, and blue dots are tRNAs, rRNAs, catalytic RNAs, and uncategorized RNAs, respectively.

The dotted lines represent the 5th and 95th percentiles of the distribution. All RNA molecules (except for PDB ID 1ZDJ, a single-stranded viral RNA) fall below the lower 5th percentile. Therefore, $99.9 \%$ of RNA structures (including ribosomal RNA) exhibit base-normal orientations that are either coplanar or bipolar. A list of all the PDB structures tested, along with their corresponding coplanarity score and barycentric coordinates are provided in the Supplemental Data.

\section{Statistical evaluation of base-normal coplanarity of RNA structural elements}

To further evaluate the degree of coplanarity in RNA structure, we performed an analysis of individual structural elements across many RNA structures. Figure 5, A and D plots the results of this analysis for helices, hairpin loops, junctions, and single-stranded regions of RNA, respectively. Figure 5A clearly illustrates the bipolar distribution of helical base-normal vectors, with a majority of the struc- tural density occurring in the bottom left-hand portion of the triangle. Figure $5 \mathrm{~B}$ suggests that the bases in most RNA hairpins also adopt generally bipolar orientations, although it is clear that there is far more scatter in the data. Similar conclusions can be drawn from the inspection of Figure 5, C and D.

We applied the same simulationbased statistical test as above to evaluate the coplanar nature of the distributions of the different structural element basenormal vectors; the results are summarized in Table 1. Not surprisingly, a majority of helices in RNA (94\%) exhibit clear bipolar orientations of their base-normal vectors. The $6 \%$ of helices that have base-normal vectors that are not significantly bipolar or coplanar are all short (six bases or less). Our statistical criteria are sufficiently stringent that small perturbations in short helix structure reduce the significance of the test, as there is a high probability for six randomly oriented vectors to appear coplanar or bipolar under the null hypothesis. Interestingly, only about $47 \%$ and $43 \%$ of hairpin loops and junctions are statistically coplanar or bipolar, respectively. These low percentages are also due to the generally small number (4-10) of bases in most RNA hairpin loops and junctions. The least coplanar or bipolar elements of RNA structure are, not surprisingly, singlestranded regions.

We are also interested in whether the coplanarity observed in hairpins and junctions extends to the neighboring helices. We therefore performed coplanarity tests on the hairpins and junctions that were found to be planar by extending the $5^{\prime}$ and $3^{\prime}$ strands by two bases. We repeated the planarity tests on these extended structures and determined that $81 \%$ of hairpins are coplanar with their neighboring helices, while only $22 \%$ of junctions exhibit extended coplanarity.

\section{Statistical evaluation of coaxial RNA helical arrangement}

The high percentage of helices exhibiting statistically significant bipolar arrangements of their base-normal vectors enables us to define the mean axis of a helix (a precise description of bipolarity is given in the Materials and Methods section). For each RNA molecule it is therefore possible to evaluate the extent to which helices are arranged 

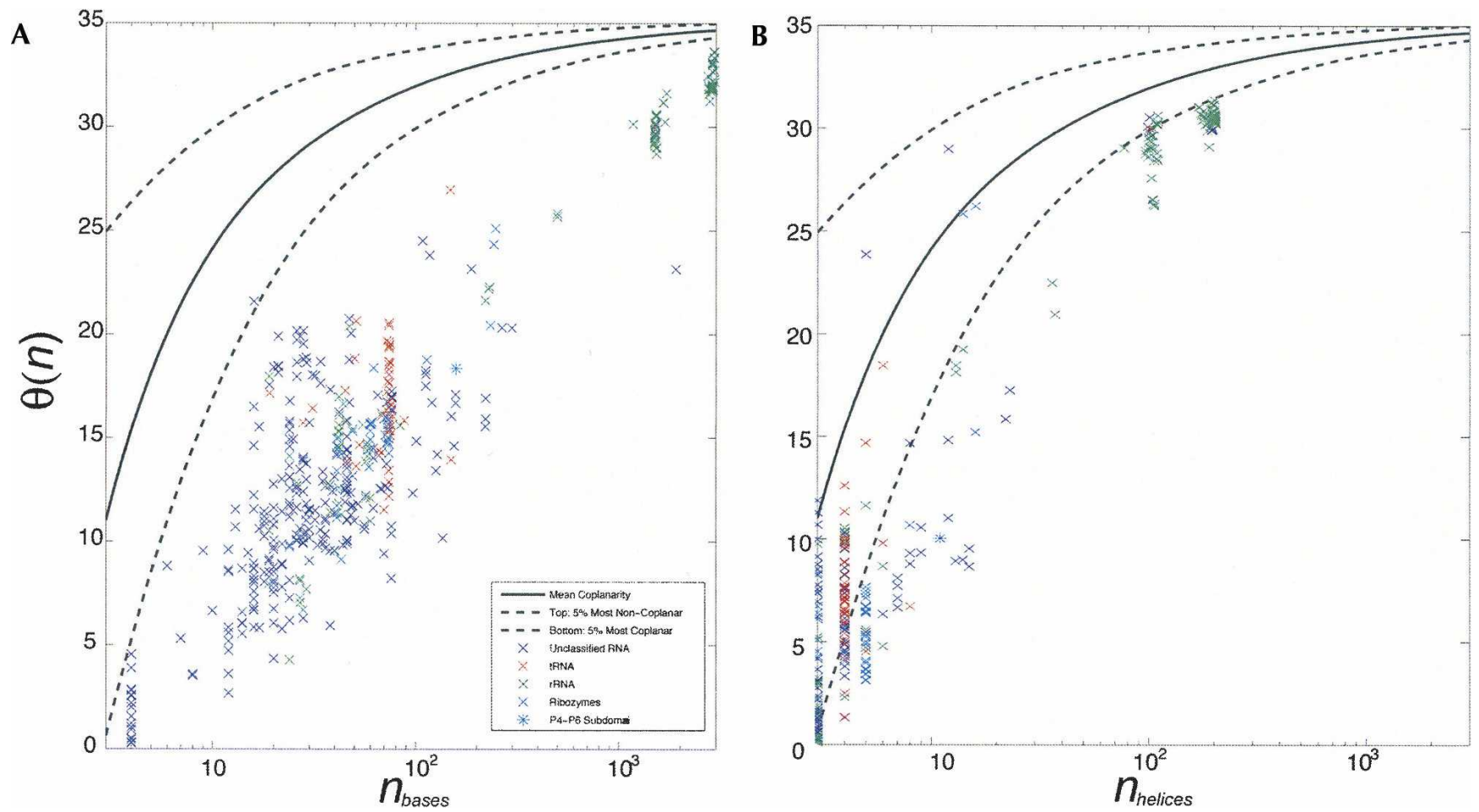

FIGURE 4. (A) Illustration of the statistical test for coplanarity or bipolarity in the distribution of base-normal vectors for all known RNAs. The expected average angular dispersion $(\theta[n])$ is plotted as a black line (for randomly generated vector orientations) while the two dotted lines indicate 95\% confidence intervals. The measured average angular dispersion for each RNA is plotted as a cross (red, green, cyan, and blue are tRNAs, rRNAs, catalytic RNAs, and uncategorized RNAs, respectively). Three hundred thirty out of 331 (99.9\%) of the crosses fall below the $95 \%$ confidence interval. (B) Same as in A, but the distribution of aggregate helical mean-axis vectors are being tested. Sixty-two percent (208/331) of the RNA structures exhibit statistically significant global coaxial arrangements of helices.

with a coplanar orientation of their axes. We therefore analyzed the distributions of the mean axes of helices for each RNA molecule in our test set and performed a test of significance with $p<0.05$. The results of this test are illustrated in Figure 4B, and interestingly a majority of RNA still falls below the 5\% percentile, illustrated as a dashed line. Of the molecules tested, $62 \%$ (false discovery rate $[\mathrm{FDR}]=0.05)$ of RNA structures show significant coaxial arrangement of helices, including most of the ribosomal RNAs (Fig. 4B, plotted in green). These results demonstrate that helices are generally arranged coaxially in large RNAs independent of size.

\section{DISCUSSION}

Compared to proteins, RNA molecules are composed of fewer and chemically less-diverse monomers. Given this apparent diminished potential for diversity, it is surprising how diverse are the functions carried out in the cell by folded RNA (Doherty and Doudna 2001). The chemical nature of the RNA residue (sugar-phosphate backbone attached to a purine or pyrimidine base) suggests that sequential stacking for bases is energetically favorable, as it is the only orientation that effectively shields the hydro- phobic faces of the bases from solvent (Ralston et al. 2000). The dominance of the A-form helix in RNA structures attests to the stability of stacked conformations in RNA.

Our results confirm statistically the overall importance of stacking in RNA structure. They also suggest a subtler general tendency in structured RNA: the coplanar or even bipolar orientation of base normals. The chemical origin of this observation is not apparent when one considers only single bases. This emergent behavior in RNA structure is therefore a consequence of the overall fold of the molecule. We, and others (Duckett et al. 1997), propose that this is a consequence of the local stereochemistry in doublestranded, nonhelical regions (or two-way junctions). The energetic advantages of base stacking in junctions favor these types of orientations. Furthermore, metal-ion binding in junctions has been shown to favor these types of orientations as well (Duckett et al. 1997). Our work demonstrates that this behavior is general, even in very large RNAs such as the ribosome.

The orientation not only of base normals but also of helix axes and (to a slightly lesser extent) the normals of bases involved in other RNA structural elements may not be totally surprising. The great stability of base-stacking interactions derives substantially from the delocalization 

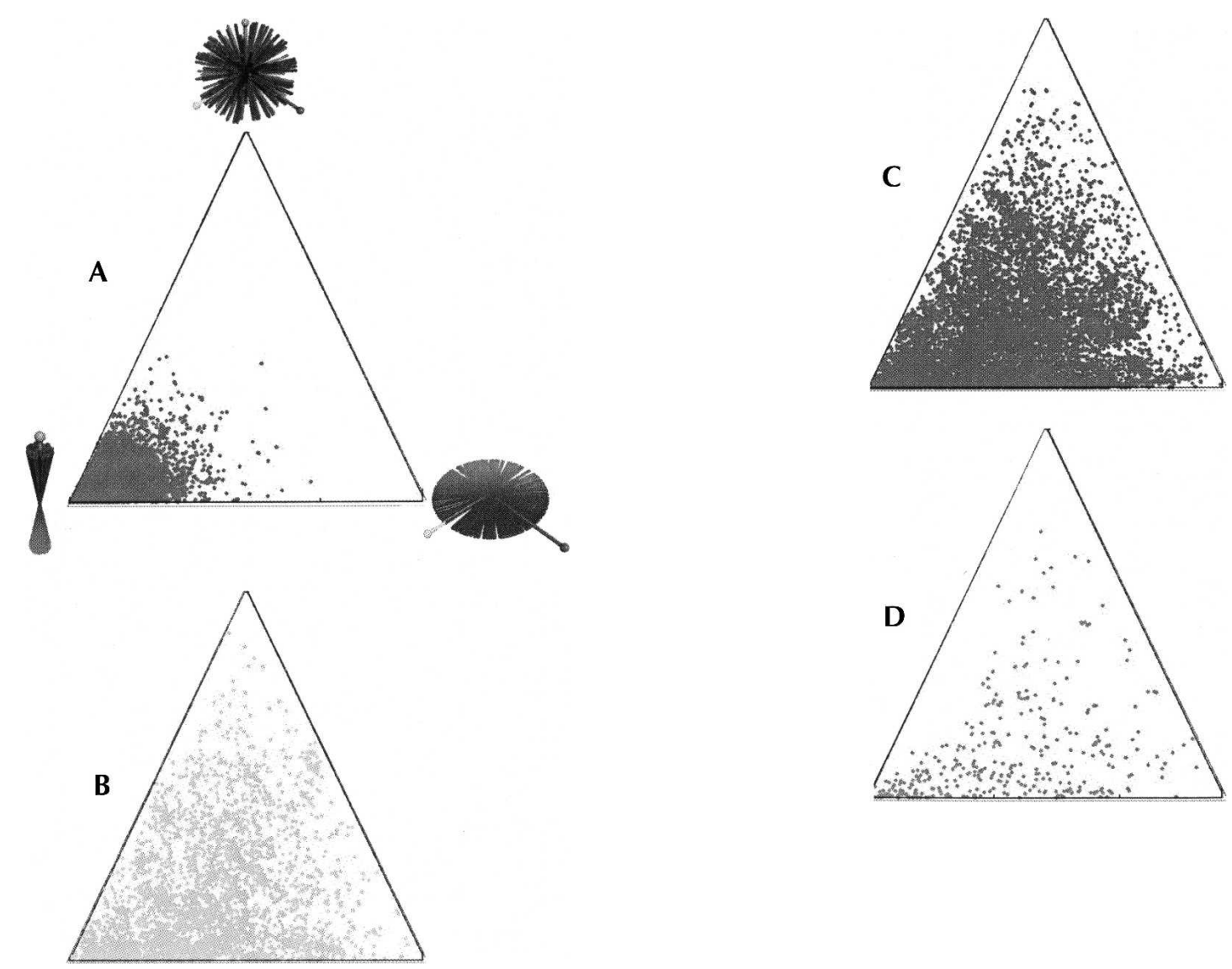

FIGURE 5. (A) Plot of barycentric coordinates for all known RNA helices as defined by Equation (2), demonstrating that most RNA helices are bipolar. $(B-D)$ Corresponding plots for hairpins, junctions, and single-stranded regions, respectively.

of electrons in the ring systems of bases. The increasingly large database of three-dimensional structures has shown that stacking interactions not only occur within the A-form double-stranded helix, but also often occur opportunistically across structural elements in an RNA. These opportunistic stacking interactions seem to create a network of connections between structural elements. Our results show that the effect of these interactions is to create larger scale "coherence" in the orientation of structural elements within large RNA structures that might otherwise be expected to show "randomized" orientations.

The use of the scatter matrix (S) and derived parameters such as the angular dispersion allowed us to define a stringent statistical test to measure coplanarity and bipolarity in RNA base-normal distributions. We found that in 99.9\% of known RNA structures, the orientations of basenormal vectors are significantly coplanar $(p<0.05)$. More surprisingly, in $62 \%$ (FDR $=0.08)$ of known RNA structures, the orientations of the coaxial vectors are also coplanar. This result suggests that the local effects observed in the stereochemistry of junctions generally affect the global fold of the RNA. Therefore, understanding local structural effects in RNA is key to predicting global fold.
Our work is a basic observation of an RNA structural phenomenon. It suggests that a good first guess of the optimal orientation for visualization of an RNA molecule can be computed as the eigenvector corresponding to the smallest eigenvalue of the scatter matrix (S) as defined in Equation (1) below. We plan to incorporate this feature in a future release of our RNA specific visualization software toRNAdo (https://simtk.org/home/rna-viz-proto). Furthermore, when modeling RNA structurally, the general tendency of helices to pack coaxially can be used to refine models further. It is therefore important to evaluate the

\begin{tabular}{|c|c|c|c|c|}
\hline & $\begin{array}{c}\text { Significantly } \\
\text { planar }\end{array}$ & Total & Percentage & $\begin{array}{c}\text { False } \\
\text { discovery } \\
\text { rate }\end{array}$ \\
\hline Helix & 17,643 & 18,831 & $94 \%$ & 0.05 \\
\hline Hairpin & 2012 & 4260 & $47 \%$ & 0.11 \\
\hline Junction & 4559 & 10,589 & $43 \%$ & 0.12 \\
\hline Single strand & 77 & 252 & $31 \%$ & 0.16 \\
\hline
\end{tabular}


general nature of simple observations of RNA structure to achieve higher predictive ability.

\section{MATERIALS AND METHODS}

\section{Directional statistics for RNA normal vectors}

The orientation of a plane in space is determined by a unit vector that is normal to that plane. We define the normal to the plane of each base as the cross product of the C2-C4-C6 vectors for purines and pyrimidines. The base orientations in an RNA molecule can be reduced to a collection of unit vectors in threedimensional space as illustrated in Figures 1, E and F, and 2.

We denote by $\mathbf{x}_{1}, \ldots, \mathbf{x}_{N}$ the collection of observed normal vectors to each of the $N$ bases. These vectors can be seen as points on the unit sphere (Fig. 2A). The dispersion of these points on the sphere can be described by the scatter matrix $\mathbf{S}$, defined by (Watson 1965; Mardia and Jupp 2000)

$$
\mathbf{S}=\frac{1}{N} \sum_{i=1}^{N} \mathbf{x}_{i} \mathbf{x}_{i}^{T}
$$

Notice that $\mathbf{S}$ does not change if any base plane is represented by the normal vector $-\mathbf{x}$ instead $\mathbf{x}$. The shape of the distribution on the sphere regardless of spatial orientation is described by the three eigenvalues $\lambda_{1}, \lambda_{2}$, and $\lambda_{3}$ of the scatter matrix $S, \lambda_{1}$ being the largest and $\lambda_{3}$ the smallest. If $\lambda_{1}$ is predominantly larger than $\lambda_{2}$ and $\lambda_{3}$, then the distribution is mostly bipolar and its mean direction is given by the eigenvector corresponding to $\lambda_{1}$. If $\lambda_{3}$ is predominantly smaller than $\lambda_{1}$ and $\lambda_{2}$, then the distribution is mostly planar and its mean plane is orthogonal to the eigenvector corresponding to $\lambda_{3}$.

We are interested in the degree to which the ensemble of vectors is coplanar, bipolar, or random. For this we further define the following three parameters (Zhang et al. 2004):

$$
\begin{aligned}
& c_{b}=\frac{\lambda_{1}-\lambda_{2}}{\lambda_{1}+\lambda_{2}+\lambda_{3}} \\
& c_{p}=\frac{2\left(\lambda_{2}-\lambda_{3}\right)}{\lambda_{1}+\lambda_{2}+\lambda_{3}} \\
& c_{r}=\frac{3 \lambda_{3}}{\lambda_{1}+\lambda_{2}+\lambda_{3}}
\end{aligned}
$$

The three parameters $\left(c_{b}, c_{p}\right.$, and $\left.c_{r}\right)$ specify the bipolar, planar, and random (or spherical) arrangement for a given distribution of vectors. Notice that each of the parameters takes values between 0 and 1 and that the three quantities add up to 1 . By plotting these three $\left(c_{b}, c_{p}\right.$, and $\left.c_{r}\right)$ values on barycentric coordinates, the space of all possible anisotropies can easily be visualized.

\section{Statistical evaluation of planarity through simulation}

The null hypothesis of our statistical test (the one we wish to reject) is that RNA base-normal vectors are uniformly and randomly distributed on a sphere. The alternative hypothesis is that the base-normal vectors are arranged on a plane. To test this hypothesis we use as a test statistic the angular dispersion $\theta$, a measure of the average rise or deviation of the vectors from the best fitting plane. The angular dispersion is defined as (Schwartzman et al. 2005)

$$
\theta=\arcsin \left(\sqrt{\lambda_{3}}\right)
$$

where $\lambda_{3}$ is the smallest eigenvalue of the scatter matrix $S$ (Equation [1]). The test is a one-sided test that rejects the null hypothesis at level 0.05 if $\theta$ is smaller than the 5 th percentile of the distribution of $\theta$ under the null.

The distribution of $\theta$ under the null is unknown. We therefore adopt a simulation-based approach, whereby we generate ensembles of unit vectors with random orientations. Moreover, the likelihood of a particular set of base-normal vectors appearing planar under the null hypothesis depends on the number of bases - the smaller the number, the higher the likelihood. For this reason it is necessary to evaluate the distribution of the angular dispersion as a function of the number of vectors.

We computationally generate $10^{5}$ distinct ensembles for $n=$ $4-10^{4}$ unit vectors. For each distinct ensemble and each $n$, the average angular dispersion $\theta$ is computed using Equation (3). The mean and distribution of $\theta$ for each $n$ is parametrized in this way. For example, the resulting mean average angular dispersion $(\theta[n])$ for all 331 RNA molecules is plotted as a solid line in Figure 4. The 5th and 95th percentiles of the distribution of $\theta(n)$ are plotted as dotted lines. Finally, for each RNA structure, the null hypothesis is rejected at level 0.05 if $\theta$ is smaller than the 5 th percentile of the simulated distribution.

\section{Statistical evaluation of bipolarity through simulation}

We use the same planarity test as described above to evaluate bipolar arrangements, i.e., arrangements of vectors that lie mainly along a central axis. Since a straight line is a subset of a plane, the bipolar arrangement is a special case of arrangement that is detected by the planarity test. The planarity test is not the most powerful for detecting bipolarity (a more powerful test would be based on $\lambda_{1}$ instead of $\lambda_{3}$ ), but it suffices for our purposes.

\section{Statistical evaluation of coaxial RNA helical arrangement}

We use the same planarity test as described above to evaluate the extent to which helices are arranged with a coplanar orientation of their axes. For this test, the units are the helical means instead of individual base pairs. The mean axis of a helix is given by the eigenvector corresponding to the largest eigenvalue of the scatter matrix $\mathbf{S}$ (Equation [1], with the $\mathbf{x}_{\mathbf{i}}$ being the helical basenormal vectors).

\section{Multiple testing considerations}

In all of the above analyses, many tests were performed simultaneously. In the analyses of base-normal vectors and coaxial RNA helical arrangement, 331 tests were performed (one for each RNA molecule). The number of statistical tests used to evaluate the base-normal coplanarity of RNA structural elements is indicated in the second column of Table 1. It is well known in statistics that 
multiple testing affects significance levels. The multiple testing problem was taken into account here by assessing the false discovery rate. The FDR is the fraction of positive test results that are expected to be false positives (false discoveries). Since every test here was performed at a fixed significance level of 0.05 , an appropriate conservative estimate of FDR is 0.05 divided by the fraction of positive test results. Computed this way, the FDR is 0.05 in the analysis of base-normal vectors, 0.08 in the evaluation of coaxial RNA helical arrangement, and corresponding numbers in the evaluation of base-normal coplanarity of RNA structural elements as indicated in the fourth column of Table 1. The interpretation is that only [1-FDR] of the declared coplanar structures (e.g., only $0.89 \times 47 \%=42 \%$ of hairpin structures) are true findings. However, the identity of the false-positive structures cannot be determined.

\section{Informatics for RNA structural analysis}

Annotation of RNA secondary structure elements was carried out using RNAView (Yang et al. 2003). A digital object model (DOM) of the structural annotations was built based on the RNAML (Waugh et al. 2002) output of RNAView and used to identify the different structural elements (helices, junctions, hairpin loops, and single-stranded regions) of the structure. The software developed to do this analysis (rnaDB.py) is freely available for download from https://simtk.org/home/rnadb/. Statistical tests and simulations were carried out in MATLAB and Python. Structures of RNA were downloaded from the Nucleic Acid Database (Berman et al. 2003). Only crystal structures with more than four helices were considered for the statistical tests.

\section{SUPPLEMENTAL DATA}

Supplementary Material and the RNADB software can be found under the download link at https://simtk.org/home/rnadb.

\section{ACKNOWLEDGMENTS}

This work was funded by NIH grant NIGMS P01-GM66275 and NIH Roadmap grant U54 GM072970 for the National Centers for Biomedical Computation. A.L. is funded by a Damon Runyan Cancer Research Foundation post-doctoral fellowship. J.M.C. is funded by a Stanford University undergraduate fellowship. A.S. is funded by a William R. and Sara Hart Kimball Stanford Graduate Fellowship and the Dana-Farber Cancer Institute.

\section{Received November 10, 2006; accepted January 24, 2007.}

\section{REFERENCES}

Auffinger, P. and Westhof, E. 2001. An extended structural signature for the tRNA anticodon loop. RNA 7: 334-341.

Berman, H.M., Westbrook, J., Feng, Z., Iype, L., Schneider, B., and Zardecki, C. 2003. The nucleic acid database. Methods Biochem. Anal. 44: 199-216.

Cate, J.H., Gooding, A.R., Podell, E., Zhou, K., Golden, B.L., Kundrot, C.E., Cech, T.R., and Doudna, J.A. 1996. Crystal structure of a group I ribozyme domain: Principles of RNA packing. Science 273: 1678-1685.

Cech, T.R. 1990. Self-splicing of group I introns. Annu. Rev. Biochem. 59: 543-568.

Doherty, E.A. and Doudna, J.A. 2001. Ribozyme structures and mechanisms. Annu. Rev. Biophys. Biomol. Struct. 30: 457-475.

Duckett, D.R., Murchie, A.I., Clegg, R.M., Bassi, G.S., GiraudPanis, M.J., and Lilley, D.M. 1997. Nucleic acid structure and recognition. Biophys. Chem. 68: 53-62.

Koradi, R., Billeter, M., and Wuthrich, K. 1996. MOLMOL: A program for display and analysis of macromolecular structures. J. Mol. Graph. 14: 51-55.

Lemieux, S. and Major, F. 2002. RNA canonical and noncanonical base-pairing types: A recognition method and complete repertoire. Nucleic Acids Res. 30: 4250-4263.

Leontis, N.B. and Westhof, E. 2003. Analysis of RNA motifs. Curr. Opin. Struct. Biol. 13: 300-308.

Leontis, N.B., Hills, M.T., Piotto, M., Ouporov, I.V., Malhotra, A., and Gorenstein, D.G. 1995. Helical stacking in DNA three-way junctions containing two unpaired pyrimidines: Proton NMR studies. Biophys. J. 68: 251-265.

Leontis, N.B., Stombaugh, J., and Westhof, E. 2002a. Motif prediction in ribosomal RNAs: Lessons and prospects for automated motif prediction in homologous RNA molecules. Biochimie 84: 961-973.

Leontis, N.B., Stombaugh, J., and Westhof, E. 2002b. The nonWatson-Crick base pairs and their associated isostericity matrices. Nucleic Acids Res. 30: 3497-3531.

Leontis, N.B., Altman, R.B., Berman, H.M., Brenner, S.E., Brown, J.W., Engelke, D.R., Harvey, S.C., Holbrook, S.R., Jossinet, F., Lewis, S.E., et al. 2006a. The RNA Ontology Consortium: An open invitation to the RNA community. RNA 12: 533541.

Leontis, N.B., Lescoute, A., and Westhof, E. 2006b. The building blocks and motifs of RNA architecture. Curr. Opin. Struct. Biol. 16: 279-287.

Lescoute, A., Leontis, N.B., Massire, C., and Westhof, E. 2005. Recurrent structural RNA motifs, isostericity matrices, and sequence alignments. Nucleic Acids Res. 33: 2395-2409.

Mardia, K.V. and Jupp, P. 2000. Directional statistics. Wiley, New York.

Ralston, C.Y., He, Q., Brenowitz, M., and Chance, M.R. 2000. Stability and cooperativity of individual tertiary contacts in RNA revealed through chemical denaturation. Nat. Struct. Biol. 7: 371-374.

Schroeder, R., Barta, A., and Semrad, K. 2004. Strategies for RNA folding and assembly. Nat. Rev. Mol. Cell Biol. 5: 908-919.

Schwartzman, R.F., Dougherty, R.F., and Taylor, J.E. 2005. Crosssubject comparison of principal diffusion direction maps. Magn. Reson. Med. 53: 1423-1431.

Tucker, B.J. and Breaker, R.R. 2005. Riboswitches as versatile gene control elements. Curr. Opin. Struct. Biol. 15: 342-348.

Watson, G.S. 1965. Equatorial distributions on a sphere. Biometrika 52: 193-201.

Waugh, A., Gendron, P., Altman, R., Brown, J.W., Case, D., Gautheret, D., Harvey, S.C., Leontis, N., Westbrook, J., Westhof, E., et al. 2002. RNAML: A standard syntax for exchanging RNA information. RNA 8: 707-717.

Yang, H., Jossinet, F., Leontis, N., Chen, L., Westbrook, J., Berman, H., and Westhof, E. 2003. Tools for the automatic identification and classification of RNA base pairs. Nucleic Acids Res. 31: 3450-3460.

Zhang, S., Bastin, M.E., Laidlaw, D.H., Sinha, S., Armitage, P.A., and Deisboeck, T.S. 2004. Visualization and analysis of white matter structural asymmetry in diffusion tensor MRI data. Magn. Reson. Med. 51: 140-147. 

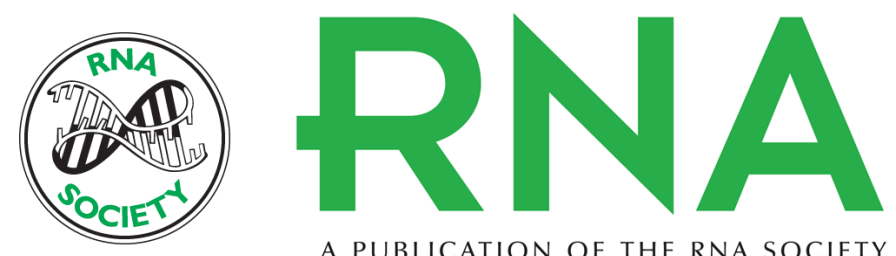

A PUBLICATION OF THE RNA SOCIETY

\section{Coplanar and coaxial orientations of RNA bases and helices}

Alain Laederach, Joseph M. Chan, Armin Schwartzman, et al.

RNA 2007 13: 643-650 originally published online March 5, 2007

Access the most recent version at doi:10.1261/rna.381407

\section{References This article cites 23 articles, 4 of which can be accessed free at: http://rnajournal.cshlp.org/content/13/5/643.full.html\#ref-list-1}

\section{License}

Email Alerting Receive free email alerts when new articles cite this article - sign up in the box at the Service top right corner of the article or click here.

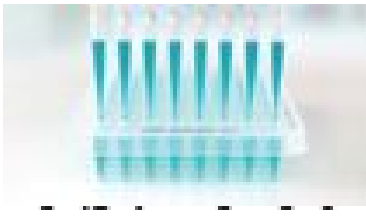

